\title{
Key Process and Quality Characteristic Identification for Manufacturing Systems using Dynamic Weighting Function and D-S Evidence Theory
}

\author{
Qingwen Yuan ${ }^{\mathrm{a}}$, Shun Jia ${ }^{\mathrm{b}, \mathrm{c}, \mathrm{*}}$, Qinghe Yuan ${ }^{\mathrm{b}, \mathrm{c}, \mathrm{d}}$, Zhaojun $\mathrm{Li}^{\mathrm{e}}$, and Xianhui Yin ${ }^{\mathrm{f}}$ \\ ${ }^{a}$ Department of Finance and Economics, Shandong University of Science and Technology, Jinan, 250031, China \\ ${ }^{b}$ Department of Industrial Engineering, Shandong University of Science and Technology, Qingdao, 266590, China \\ ${ }^{c}$ State Key Laboratory of Mining Disaster Prevention and Control, Shandong University of Science and Technology, Qingdao, 266590, China \\ ${ }^{d}$ National Demonstration Center for Experimental Mining Engineering Education (Shandong University of Science and Technology), \\ Qingdao, 266590, China \\ ${ }^{e}$ Department of Industrial Engineering and Engineering Management, Western New England University, Springfield, 01119, USA \\ ${ }^{f}$ College of Management and Economics, Tianjin University, Tianjin, 300072, China
}

\begin{abstract}
Monitoring and controlling of process and quality characteristic for manufacturing system is a key issue in closed-loop quality control. Meanwhile, it is challenging due to the modern manufacturing system generally consists of hundreds of processes that are aligned to produce a specific end product. And the output quality of each individual process may be judged by dimensional quality characteristics. Each process and quality characteristic being monitored is costly and impractical. This paper attempts to provide a systematic approach to identify the key processes and quality characteristics simultaneously. Firstly, a modified casual matrix is used to acquire the correlation data between process and characteristic. The correlation degree is weighted by dynamic weighting function based on the importance of quality characteristics. Then, the triangular fuzzy function is used to construct the frame of discernment based on single index (quality characteristic). The mass functions that represent the degree of belief supported are determined and treated as pieces of evidence. Afterward, all of the evidence are combined by D-S (Dempster-Shafer) fusion rules. In addition, key quality characteristics are also identified based on the cumulative sum of the weighted score and Pareto Principle simultaneously. Finally, the usefulness of proposed approach is verified by a real-time dense medium coal preparation case.
\end{abstract}

Keywords: key process and quality characteristic; quality control; fuzzy function; evidence theory; coal preparation system

(Submitted on May 13, 2018; Revised on June 25, 2018; Accepted on July 25, 2018)

(C) 2018 Totem Publisher, Inc. All rights reserved.

\section{Introduction}

Monitoring and controlling of production process/quality characteristic is a current way to assure the product quality. However, with the advancement of technology, the configuration of the production system becomes more and more sophisticated. A modern manufacturing system generally consists of hundreds of processes that are aligned sequentially to produce a specific end product. All processes being monitored are unreasonable and costly. In addition, there are multiple dimensions for quality characteristic (e.g., product quality characteristics and process quality characteristics). Product quality characteristic mainly reflects the attributes of product, which means the parameters focus on production process that are not considered in quality monitor and control. Each dimension of quality characteristic generally includes many quality characteristics. Thus, it is crucial for practitioners that some processes and characteristics are identified as key processes and quality characteristics to give prior control. The essence of key processes and quality characteristic is a problem of cluster and decision making. Evaluation indicators, correlation function between process and classification and decision making rule play an important role in solving the problem. Conventional approaches for key process and characteristic are based on quality function development (QFD) and Kano model [1-4]. The quality characteristics from customers' concern are designed and assured in production system. The processes consisting of identified key quality characteristic are also identified as key processes [5-8]. However, the identified key processes and quality characteristics solely depend on product

\footnotetext{
* Corresponding author.

E-mail address: herojiashun@163.com
} 
quality characteristics, which ignore the influence of some key process parameters. Therefore, these conventional works cannot satisfy the requirements of internal customer.

In addition to these traditional methods, there are extensive research works being reported on identification of key process and quality characteristic. The existing literature on key process and characteristic identification can be classified into two broad categories: viz. bottle-neck stage/process identification and key quality characteristics identification.

From the perspective of bottleneck identification, Kamil et al. [9] proposed a bottleneck identification approach in supply chain networks (SCN). They demonstrate that some methods based on network theory may support the bottleneck identification process. However, they can also be misleading because they often focus only on a small part of the network or have been developed for a particular application, not for supply chain risk management. Therefore, they propose an alternative method of bottleneck identification that considers the features of modern SCN. It can be seen that the identification approach is presented from risk management insight of supply chain. Sujova et al. [10] insist that the key subprocess is related to the manufacturing of the key product contributing to the final value of production at most. Arumugam and Saral [11] use graph theoretic techniques to develop an efficient algorithm for identifying bottleneck elements with respect to a given solution of the cellular manufacturing problem. Yu and Matta [12] proposed a statistical framework of data-driven bottleneck identification in manufacturing system. Due to variation source identification for manufacturing processes, it is critical for product dimensional quality improvement. So, various techniques have been developed in recent years. The processes with identified variation source are always described as bottle-neck processes [13-14]. The variation source is identified as key quality characteristic to be monitored by the corresponding control chart [15]. For example, Zhou and Jiang [16] presented a systematic method of variation source identification for deep hole boring process based on multisource information fusion using Dempster-Shafter (D-S) evidence theory. The critical processes are determined simultaneously. The modeling and optimization problem of quality variation in multistage manufacturing system becomes a hot issue in recent years. Therefore, the identification and diagnosis of bottle-neck processing stages play an important role in these research works. For small-batch multistage machining processes of complex aircraft parts, Wang and Jiang [17] proposed an analytical structure of the fluctuation evaluation and identification model for small-batch multistage machining process, which comprises four levels, namely, part level, multistage level, single-stage level and quality feature level. Corresponding to the four levels in the analytical structure, four fluctuation analysis indices are proposed to quantitatively evaluate the fluctuation level of different parts and identify the weak stages and elements that result in the abnormal fluctuation in the process flow. Besides, Xu et al. [8], Du et al. [18] and Du et al. [19] developed the quality bottle-neck identification in multistage manufacturing system based on Markov model. Vandebroek et al. [20] described a simple procedure based on cluster detection to identify the problematic steps that is identified as a key step. There are some researchers developed the bottle-neck stage identification based on the steam of variation propagation theory [21-25]. In addition to bottleneck and variation source identification, the key processes identification method is directly proposed in a little research works. For instance, Tang et al. [26] developed the method based on the theory of process management and $80 / 20$ principle to determine the key processes of the workshop manufacturing processes, and the concept of criticality was presented to describe the importance of process nodes in the proposed model.

From the perspective of key quality characteristic identification, Ma et al. [27] insisted that all quality characteristics need to be constantly tested in the process of mechanical parts machining. The efficiency is low and the cost is high. Therefore, the key quality characteristics (KQCs) identification is helpful for narrowing the scope of inspection and improving the detection efficiency. Aiming at this problem, they proposed Mahalanobis-Taguchi System (MTS) method based on ReliefF algorithm. To improve the existing methods of identifying the key quality characteristics in multistage manufacturing process, an identification method for key quality characteristics in multi-stage manufacturing process was provided by combining the Partial Least Squares Regression (PLSR) method with the state space model by Wang et al. [28]. Some other relevant literature on key variable or parameter identification for quality monitor and control are discussed by Jin et al. [29], Foggia and D’ Addona [30], Li and Zheng [31] and Kim et al. [32].

Although extensive research works have been reported on identification problem of key/bottle-neck process and quality characteristic/parameter, there are certain limitations in existing literature. The key limitations of existing research on key process and quality characteristic identification are as follows:

(i) The identification of key process and quality characteristic is studied separately. This means that a framework that can identify key process and quality characteristic/parameter simultaneously is not provided in existing researches.

(ii) Most existing identification methods are not suitable for small-lot production systems due to the requirement for lots of specific production data, i.e., defects frequency and quality deviation of response characteristics. In these works, sophisticated mathematical model needs to be established. This may be difficult for some industries because of the 
backwardness of sensor technology. In addition, the information system needs to be installed to deal with the complex computing. Meanwhile, the statistical data cannot indicate the loss of failure, which means that although some processes may have low failure/defect frequency; these processes would cause enormous loss once failed.

(iii) The weights of different evaluation indicators are equal in previous researches. The evaluation indicators mainly consist of product quality characteristic, and other quality characteristics, i.e., process quality characteristic, safety quality characteristic and work quality characteristic are not considered.

(iv) There may be multiple and different decision results due to different quality characteristic dimensionality and the decision results may be conflicted. The conventional methods to deal with the problem are to give reasonable weights. But, these weights always are determined by experience.

Therefore, the objective of this research is to propose a new systematic approach in quality insights to identify key processes and quality characteristics simultaneously. The problems of static weight, sophisticated computing and multiple decision combination can be solved. To deal with the problem, this paper attempts to develop an efficient and simplified approach to identify the key processes and quality characteristics simultaneously for sophisticated production system. Firstly, a modified casual matrix is used to establish the correlation between process and characteristic. Then, raw data is provided by expert scoring. The data is weighted by dynamic weighting function according to the importance of quality characteristics. Afterward, the decision result of each process can be given based on adjusted data of individual characteristic by triangular fuzzy function and treated as pieces of evidence. Finally, the D-S evidence fusion rules are presented to synthesize the multiple decision results to identify final key processes. In addition, key characteristics are also identified based on cumulative sum of weighted score and Pareto principle.

The remainder of this paper is organized as follow. Section 2 describes the detail of proposed approach, viz. the schematic representation of the identification model, the data acquisition method based on modified casual matrix, the specific dynamic weighting fuzzy function and D-S evidence synthesis theory based key processes identification method and 80/20 principle based key quality characteristics identification method. Section 3 presents a real-life coal preparation case to demonstrate the detailed application procedure and verify the effectiveness of proposed approach. In addition, the reliability of raw data and characteristics of final decision result are discussed in this section. Section 4 gives the conclusion as well as cites possible avenues of future research direction in this field of study.

\section{Construction of Key Processes and Quality Characteristics Identification Approach}

In this section, the schematic configuration of key processes and quality characteristics identification approach, the mathematical solution formulation and application procedure of proposed approach are presented in details.

\subsection{Schematic Representation of Key Process and Quality Characteristic Identification Approach}

The proposed identification approach is a dynamic system. The inputs of the system are initial processes (characteristics) and data/information; the outputs are value and knowledge (key processes/characteristics). The mechanism/rule transforming inputs into outputs is specific computing methods or function. A schematic diagram (logic framework) of key processes and characteristics identification approach is shown in Figure 1.

As seen from Figure 1, the modified casual matrix is used to establish the correlation between processes and dimensional quality characteristics. Then, the dynamic weighted data reflecting the correlation coefficient is used and analysed based on specific mathematical solution formulation to identify the key processes and characteristic.

\subsection{Data Acquisition}

This section is divided into two subsections. The first subsection presents the modified casual matrix to establish the correlation between processes and quality characteristics. The second subsection describes the data acquisition method.

\subsubsection{Establishment of Correlation Between Process and Quality Characteristic}

The proposed approach is designed to identify key processes from quality insights. Therefore, the establishment of correlation between processes and quality characteristics is critical for this work. Causal matrix, which is also called characteristics selection matrix, is a tool that can help determine the priority of importance for the key process inputs [33]. Application of causal matrix is to determine the key input variables that have the largest degree of influence on the outputs through building a correlation between input variables and output variables. 
The conventional causal matrix model reflects the degree of correlation between input variables and output variables. However, in our research work, we need to establish the relation between processes and quality characteristics. Therefore, we redesigned the input variables and output variables of the model, called modified casual matrix. The processes are treated as input variables and the quality characteristics are served as output variables in modified casual matrix. The correlation coefficient presents the influence degree of various processes on multiple quality characteristics. The modified casual matrix is shown in Figure 2.

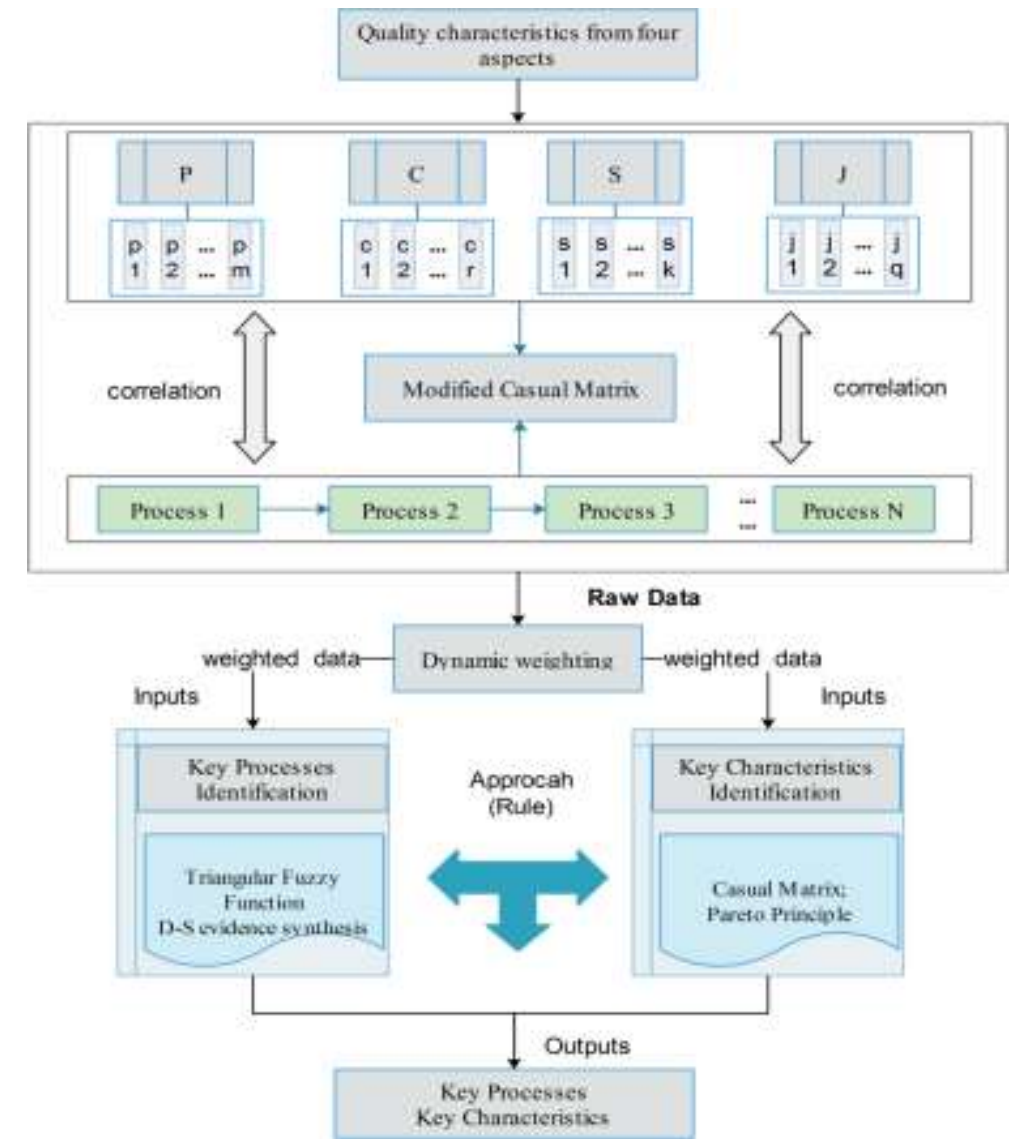

Figure 1. Schematic diagram of key processes and characteristics identification approach

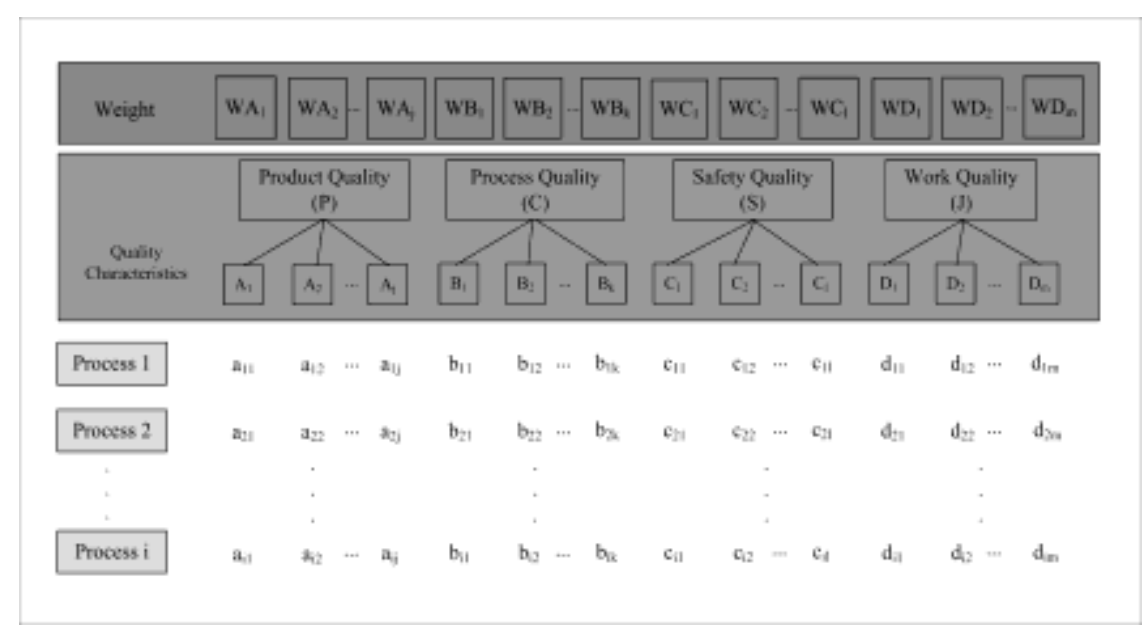

Figure 2. The modified casual matrix

It can be seen from Figure 2 that the quality characteristics consist of multiple dimensions characteristics. According to Total Quality Management (TQM) theory, the quality characteristics can be divided into product quality, process quality, safety quality, and work quality [34-36]. Among them, product quality shows whether the quality of the product 
could satisfy the demands of the customer or enterprise. Process quality is the quality of equipment, materials, technologies invested to achieve the quality control of the production process. Safety quality is a series of problems of safety assurance degree. Work quality is the quality of application of technology and management for ensuring product quality, process quality and safety quality. Besides, in the multiple characteristics context, each quality characteristic may have a different weight.

\subsubsection{Data Acquisition}

In this work, Experts Grading Method (EGM) is presented to assess the correlation between processes and characteristics [37-38]. The detailed scoring criterion is provided in Table 1.

Table 1. The scoring criterion

\begin{tabular}{|c|c|c|c|}
\hline Level & Score & Level definition & Converted score \\
\hline 0 & 0 & No correlation & 0 \\
\hline I & $1,2,3$ & Slightly correlation between process and quality characteristic & $0.1,0.2,0.3$ \\
\hline II & $4,5,6$ & Some impact of process on quality characteristic & $0.4,0.5,0.6$ \\
\hline III & 7,8 & A more significant impact of process on quality characteristic & $0.7,0.8$ \\
\hline IV & 9,10 & A significant impact of process on quality characteristic & $0.9,1.0$ \\
\hline
\end{tabular}

As seen from Table 1, Ten-Point System (TPS) is used in this scoring criterion. I IV level corresponds to 1-10 points. No correlation is presented by 0 . To analyze and derive the dynamic weight of quality characteristics, the score is converted to $0 \sim 1.0$.

The effectiveness and practicability of scoring data is important for key processes identification. To assure the effectiveness of data, the scoring subject includes three parts. The first scoring subject is senior managers, i.e., manager and vice manager. The second scoring subject is ordinary management personnel. The last subject is operator on site. Different weights are assigned to various scoring subjects. Therefore, the values of correlation between processes and quality characteristics are weighted sum of score from three subjects.

\subsection{Dynamic Weighting Fuzzy Function and D-S Theory based Key Processes Identification}

In this section, dynamic weighting formulation of each quality characteristic, fuzzy membership function used to determine the degree of membership between weighted score and grade of process, and D-S evidence synthesis based rules are presented in details.

As seen from Table1, the same scoring level has different scores. In other words, not only is there multiple grades/levels scoring criterion, but also a difference in the same grade. Therefore, the constant/static weight assignment method is unreasonable to determine the weight of various quality characteristics. In this work, the dynamic weighting function is provided to determine the weight of characteristic. For each process, the dynamic weighting function is shown as Equation (1).

$$
\omega_{j}(x)=\left\{\begin{array}{l}
0, x \leq \beta_{j} \\
1-e^{-\left(\frac{x-\beta_{j}}{\sigma_{j}}\right)}, x>\beta_{j}
\end{array}\right.
$$

Where $\omega_{j}(x)$ is the dynamic weight of $j^{\text {th }}$ quality characteristic. $\beta_{j}$ represents the median of first (I) scoring level. $\sigma_{j}$ can be calculated by Equation (2).

$$
\sigma_{j}=\omega_{j}\left(a_{4}^{j}\right)=0.9,1 \leq j \leq M
$$

In the above equations, $\mathrm{M}$ is the number of quality characteristics, and $a_{4}^{j}$ represents the lower value of fourth (IV) scoring level on $j^{\text {th }}$ quality characteristic.

According to the data from modified casual matrix and dynamic weighting function, the degree of correlation between each process and each characteristic can be calculated as Equation (3).

$$
x_{i j}^{\prime}=\omega_{i}\left(x_{i j}\right) \times x_{i j}, 1 \leq i \leq N, 1 \leq j \leq M
$$


Where $x_{i j}$ is the weighted score (degree of correlation) of three scoring subjects, $N$ is the number of processes, and $x_{i j}^{\prime}$ represents the adjusted degree of correlation between $i^{\text {th }}$ process and $j^{\text {th }}$ quality characteristic.

To derive the degree of membership of a process belongs to key process, extremum difference method is used to normalize $x_{i j}^{\prime}$. $x_{i j}^{\prime \prime}$ represents the normalized value of $x_{i j}^{\prime}$.

The production processes can be divided into four types according to related research: key process, more important process, important process and general process. The Triangular Fuzzy Function (TFF) is given to derive the degree of membership between process and four process types. The membership function is shown as Equation (4).

$$
\begin{aligned}
& u_{1}(\lambda)=\left\{\begin{array}{l}
1, \lambda=1 \\
\frac{\lambda-0.75}{0.25}, 0.75 \leq \lambda<1 \\
0,0 \leq \lambda<0.75
\end{array}\right. \\
& u_{2}(\lambda)=\left\{\begin{array}{l}
0, \lambda=1 \\
\frac{1-\lambda}{0.25}, 0.75 \leq \lambda<1 \\
\frac{\lambda-0.5}{0.25}, 0.5 \leq \lambda<0.75 \\
0,0 \leq \lambda<0.5
\end{array}\right. \\
& u_{3}(\lambda)=\left\{\begin{array}{l}
0,0.75 \leq \lambda \leq 1 \\
\frac{0.75-\lambda}{0.25}, 0.5 \leq \lambda<0.75 \\
\frac{\lambda-0.2}{0.3}, 0.2 \leq \lambda<0.5 \\
0,0 \leq \lambda<0.2
\end{array}\right. \\
& u_{4}(\lambda)=\left\{\begin{array}{l}
0,0.5 \leq \lambda \leq 1 \\
\frac{0.5-\lambda}{0.3}, 0.2 \leq \lambda<0.5 \\
\frac{0.2-\lambda}{0.2}, 0 \leq \lambda<0.2 \\
0, \lambda=0
\end{array}\right.
\end{aligned}
$$

Where $u$ is degree of membership, and $\lambda$ represents the adjusted degree of correlation $\left(\lambda=x_{i j}^{\prime \prime}\right)$.

As seen from Equation (4), there are four values of degree of membership for each $x_{i j}^{\prime \prime}$ and the largest value indicates the type/grade of the process. It means that each quality characteristic can be used to make a decision to determine corresponding key processes. Therefore, there may be multiple and different decision results. To give a final decision, the feasible and reasonable decision synthesis approach is necessary. In this work, D-S evidence synthesis rule is provided to make a final decision according to various decisions. Evidence combination is the core of key process identification based on evidence reasoning. A synthetic belief assignment function can be produced by fusing the evidence derived in mass function.

The process type is represented by a set $\Theta$, called Frame of Discernment. For each process, $\Theta=($ key process, more important process, important process, general process). For $\forall A \subseteq \Theta$, based on Dempster's combined rule, the mass function of process grade is shown as Equation (5) and Equation (6) [39-40].

$$
m(A)=\left\{\begin{array}{l}
0, A=\varnothing \\
\frac{\sum_{A_{i} \cap B_{i}=A} m_{1}\left(A_{i}\right) m_{2}\left(B_{j}\right)}{1-K}, A \neq \varnothing
\end{array}\right.
$$




$$
K=\sum_{A_{i} \cap B_{i}=A} m_{1}\left(A_{i}\right) m_{2}\left(B_{j}\right)<1
$$

Where $m$ represents basic probability function, and $m(A)$ is the basic probability of Proposition A, which reflects the support degree of Proposition A by evidences. $A_{i}$ and $B_{j}$ are initial propositions. The mass function of multiple proposition can denote as:

$$
\begin{gathered}
M=m_{1} \oplus m_{2} \oplus \cdots \oplus m_{n} \\
\operatorname{bel}(A)=M(A)
\end{gathered}
$$

Under the assumption that all process grades are independent of each other, the belief function of A can be calculated as follows.

Finally, the process type/grade of each process can be identified based on synthetic decision result $\mathrm{M}$ and maximum membership principle.

According to the above analysis, the procedure of proposed key process identification approach can be summarized in the following steps.

Step 1 Determining the quality characteristics.

Step 2 Constructing the correlation between process and quality characteristics.

Step 3 Data acquisition by scoring.

Step 4 Determining the dynamic weight of each quality characteristic and computing the weighted score of correlation.

Step 5 Computing the degree of membership between process and process grade and identifying the key process based on each quality characteristic.

Step 6 Determining the synthetic decision-making conclusions based on D-S evidence fusion rule to identify final key processes set.

\subsection{Pareto based Key Characteristics Identification}

The criticality degree $(u)$ is defined to reflect the significance of each quality characteristic. The calculation formula of $u$ of $j^{\text {th }}$ characteristic is provided in Equation (9).

$$
u_{j}=\sum_{i=1}^{N} w_{i j}\left(x_{i j}\right) \times x_{i j}=\sum_{i=1}^{N} x_{i j}^{\prime}, 1 \leq j \leq M
$$

Pareto Principle (80/20 Principle) can reveal the fact that little key factors may play a vital role for some phenomena. Therefore, the Pareto Principle is feasible to identify key quality characteristics. Firstly, the criticality degree of each characteristic is sorted according to descending order. Then, the cumulative ratio is computed. Finally, the processes with cumulative ratio to $80 \%$ are identified as key processes.

\section{Case Study}

In this section, a real-life coal preparation case is provided to demonstrate the implementation steps of key processes and characteristics identification approach. In addition, the effectiveness of proposed approach is verified. Some discussions about expert scoring and identified results also are given in this section.

\subsection{Case Backgrounds}

The dense medium coal preparation process is taken as an example. The composition of dense medium separation system could be divided into nine parts: transportation and measurement of gross coal, iron removal, screening of big-size lump coal, classified filtration, separation and dirt exclusion, dehydration and de-media, magnetic separation, recovery of concentrate and slime recovery. The nine parts could be further divided into 53 processes (shown in Table 2). 
Table 2. Dense medium separation processes

\begin{tabular}{|l|l|}
\hline No. & \multicolumn{1}{|c|}{ Processes } \\
\hline $1(\mathrm{~b})$ & Gross coal through coal feeder 100 to belt conveyer \\
\hline 2 (c) & Quantity check of belt weigher on coal feeder 100 \\
\hline $3(\mathrm{a})$ & Ironware was extracted by removal equipment of tramp iron 144 \\
\hline $4(\mathrm{~b})$ & Ironware of classification loading away \\
\hline $5(\mathrm{~b})$ & System A: the coal without ironware through the chute under belt conveyer 101 to rod vibration screen 102 \\
\hline$\vdots$ & \\
\hline 49(b) & Slime was precipitated by inclined plate settler 130 \\
\hline $50(a)$ & $\begin{array}{l}\text { Part of overflow form inclined plate settler 130 to magneto tail overflow ladle, then it was driven into thickener of main cleaning by pump 153 } \\
\text { or 154 }\end{array}$ \\
\hline $51(\mathrm{~b})$ & Slime of fine particle was recycled by thickener of main cleaning \\
\hline 52(a) & Underflow in inclined plate settler 130 was driven into the posterior segment of linear vibrating screen 108 or 109 by pump 108 or 109 \\
\hline 53(a) & Slime of crude particles was recycled by linear vibrating screen 108 or 109 \\
\hline
\end{tabular}

\subsection{Key Processes and Quality Characteristics Identification}

\subsubsection{Quality Characteristics}

According to the proposed approach, the measurement indicators of quality characteristics include four aspects, i.e., product quality, process quality, safety quality and work quality. Based on the situations of coal preparation plant, the four aspects are divided into 17 quality characteristics.

Product quality in this case is divided into 7 quality characteristics according to the national standards and customer's requirements, i.e., ash, total water of coal, refuse rate, rate of particle size small than lower size limit of lump coal, volatile, impurity content, and calorific value. Coal preparation plant is a highly mechanized and continuous production unit [41]. To ensure the uninterrupted production, process quality and product quality are also important. Process quality is divided into quantity of having medium, equipment failure rate, water spray quantity, hydraulic pressure of de-medium, qualified medium density, and inlet concentration of the magnetic separator. Among them, the quantity of having medium is mainly consisted of lump coal, gangue and slime having medium. In the processes of coal preparation, only one or less equipment exist in some processes, so that the failure of equipment may lead to stop production. Thus, equipment failure rate is a demand index of process quality. For water spray quantity and hydraulic pressure, they would influence the effect of demedium if the quantity of them is too low. On the contrary, they can't be handled sufficiently if the quantity of them is too large. In addition, the quantity has an impact on the work effectiveness of magnetic separator directly whether concentration of magnetic separator is qualified or not. The foundation of total quality management in coal preparation is safety. Safety quality is divided into three quality characteristics, i.e., the slight wounded rate, the severely wounded rate, and the death rate of employment injury. Work quality is the quality of management and technological work to improve product quality. It is divided into work efficiency and standard-reaching rate. The quality characteristics of coal preparation are shown in Table 3.

Table 3. The quality characteristics of four aspects (type)

\begin{tabular}{|c|c|}
\hline Type & Quality characteristics \\
\hline \multirow{7}{*}{ Product quality $\mathrm{P}$} & Ash (P1) \\
\hline & Total water of coal (P2) \\
\hline & Refuse rate $(\mathrm{P} 3)$ \\
\hline & Rate of particle size small than lower size limit of lump coal (P4) \\
\hline & Volatile (P5) \\
\hline & Impurity content (P6) \\
\hline & calorific value $(\mathrm{P} 7)$ \\
\hline Type & Quality characteristics \\
\hline \multirow{5}{*}{ Process quality $\mathrm{C}$} & Quantity of having medium (C1) \\
\hline & Equipment failure rate $(\mathrm{C} 2)$ \\
\hline & Water spray quantity and hydraulic pressure of de-medium (C3) \\
\hline & Qualified medium density (C4) \\
\hline & Inlet concentration of magnetic separator (C5) \\
\hline Type & Quality characteristics \\
\hline \multirow{3}{*}{ Safety quality S } & Slight wounded rate $(\mathrm{S} 1)$ \\
\hline & Severely wounded rate (S2) \\
\hline & Death rate $(\mathrm{S} 3)$ \\
\hline Type & Quality characteristics \\
\hline \multirow{2}{*}{ Work quality J } & Work efficiency (J1) \\
\hline & Standard-reaching rate $(\mathrm{J} 2)$ \\
\hline
\end{tabular}




\subsubsection{Establishment of Correlation and Data Acquisition}

As seen from section 2.2, the score of correlation is evaluated by three subjects, i.e., senior manager, ordinary management personnel and operator. Three subjects evaluate the correlation based on given scoring criterion. The score of correlation is the sum of weighted score from three subjects (According to experience, the weights of three subjects are 0.3, 0.4, 0.3). The score is shown as Table 4.

\begin{tabular}{|c|c|c|c|c|c|c|c|c|c|c|c|c|c|c|c|c|c|}
\hline Weight $(0-1)$ & $\mathrm{W}_{\mathrm{i} 1}$ & $\mathrm{~W}_{\mathrm{i} 2}$ & $\mathrm{w}_{\mathrm{i} 3}$ & $\mathrm{~W}_{\mathrm{i} 4}$ & $\mathrm{~W}_{\mathrm{i} 5}$ & $\mathrm{~W}_{\mathrm{i} 6}$ & $\mathrm{~W}_{\mathrm{i} 7}$ & $\mathrm{~W}_{\mathrm{i} 8}$ & $\mathrm{~W}_{\mathrm{i} 9}$ & $\mathrm{~W}_{\mathrm{i} 10}$ & $\mathrm{~W}_{\mathrm{i} 11}$ & $\mathrm{~W}_{\mathrm{i} 12}$ & $\mathrm{~W}_{\mathrm{i} 13}$ & $\mathrm{~W}_{\mathrm{i} 14}$ & $\mathrm{~W}_{\mathrm{i} 15}$ & $\mathrm{w}_{\mathrm{i} 16}$ & $\mathrm{~W}_{\mathrm{i} 17}$ \\
\hline NO. & 1 & 2 & 3 & 4 & 5 & 6 & 7 & 8 & 9 & 10 & 11 & 12 & 13 & 14 & 15 & 16 & 17 \\
\hline \multirow{2}{*}{ Quality characteristics (0-1) } & \multicolumn{7}{|c|}{ Product quality $\mathrm{P}$} & \multicolumn{5}{|c|}{ Process quality $\mathrm{C}$} & \multicolumn{3}{|c|}{ Safety quality $S$} & \multicolumn{2}{|c|}{ Work Quality J } \\
\hline & P1 & $\mathrm{P} 2$ & P3 & $\mathrm{P} 4$ & P5 & P6 & $\mathrm{P} 7$ & $\mathrm{C} 1$ & $\mathrm{C} 2$ & $\mathrm{C} 3$ & $\mathrm{C} 4$ & $\mathrm{C} 5$ & $\mathrm{~S} 1$ & S2 & S3 & $\mathrm{J} 1$ & $\mathrm{~J} 2$ \\
\hline \multicolumn{18}{|l|}{ Process NO. } \\
\hline 1 & 0.3 & 0.1 & 0.9 & 0.3 & 0.1 & 0 & 0 & 0.1 & 0.3 & 0.3 & 0.9 & 0.3 & 0 & 0 & 0 & 0.1 & 0 \\
\hline 2 & 0 & 0 & 0 & 0 & 0 & 0 & 0 & 0.1 & 0.3 & 0.9 & 0 & 0 & 0 & 0 & 0 & 0.1 & 0.1 \\
\hline 3 & 0 & 0 & 0 & 0 & 0 & 0 & 0 & 0.5 & 0.3 & 0.1 & 0.2 & 0.3 & 0 & 0 & 0 & 0 & 0 \\
\hline 4 & 0.1 & 0 & 0 & 0 & 0 & 0 & 0 & 0.3 & 0.6 & 0 & 0 & 0 & 0 & 0 & 0 & 0 & 0 \\
\hline$\ldots$ & $\ldots$ & $\ldots$ & $\ldots$ & $\ldots$ & $\ldots$ & $\ldots$ & $\ldots$ & $\ldots$ & $\ldots$ & $\ldots$ & $\ldots$ & $\ldots$ & $\ldots$ & $\ldots$ & $\ldots$ & $\ldots$ & $\ldots$ \\
\hline 52 & 0.5 & 0.5 & 0.9 & 0.7 & 0.3 & 0.1 & 0 & 0.1 & 0.4 & 0.3 & 0.1 & 0.3 & 0 & 0 & 0 & 0.1 & 0 \\
\hline 53 & 0.1 & 0 & 0 & 0 & 0 & 0.1 & 0 & 0.3 & 0.1 & 0.1 & 0.9 & 0.3 & 0 & 0 & 0 & 0 & 0 \\
\hline
\end{tabular}

\subsubsection{Key Processes Identification}

The parameters of dynamic weighting function $\left(\beta_{i}, \sigma_{i}\right)$ can be derived according to Equation (2). $\beta_{i}=0.1500, \sigma_{i}=0.8011, \quad($ $1 \leq i \leq 53$ ). Therefore, the dynamic weighting function is shown as Equation (10).

$$
\omega_{i}(x)=\left\{\begin{array}{l}
0, x \leq 0.1500 \\
1-e^{-\left(\frac{x-0.15}{0.8011}\right)}, x>0.1500
\end{array}\right.
$$

The adjusted scores can be acquired by Equation (3). The standardized value of adjusted (dynamics weighting) score is served as the final degree of correlation between various processes and characteristics.

The degree of membership can be computed by Equation (4). Therefore, 17 groups value of membership can be derived for each process. To reduce computing workload, the largest membership value of each quality aspect is selected as the degree of membership of this quality aspect $(\mathrm{P}, \mathrm{C}, \mathrm{S}, \mathrm{J})$. Therefore, the number of quality characteristics can reduce from 17 to 4 . The standardized values of degree of membership of four quality characteristics for $1^{\text {st }}$ process are shown in Table 5 .

Table 5. Standardized values of degree of membership for $1^{\text {st }}$ process

\begin{tabular}{|c|c|c|c|c|}
\hline Characteristics & Key process & More improtant process & Improtant process & General process \\
\hline $\mathrm{P}(1)$ & 0.8320 & 0.1160 & 0.0520 & 0.0000 \\
\hline $\mathrm{C}(2)$ & 0.4000 & 0.0000 & 0.3730 & 0.2270 \\
\hline $\mathrm{S}(3)$ & 0.0000 & 0.2820 & 0.6030 & 0.1150 \\
\hline $\mathrm{J}(4)$ & 0.3210 & 0.5320 & 0.1470 & 0.0000 \\
\hline
\end{tabular}

The confidence level set as 0.9 . The mass function values of evaluated score for $1^{\text {st }}$ process are presented in Table 6 .

Table 6. Mass function values of evaluated score for $1^{\text {st }}$ process

\begin{tabular}{|c|c|c|c|c|c|}
\hline Characteristics & Key process $\left(\boldsymbol{\theta}_{\mathbf{1}}\right)$ & More improtant process $\left(\boldsymbol{\theta}_{\mathbf{2}}\right)$ & Improtant process $\left(\boldsymbol{\theta}_{\mathbf{3}}\right)$ & General process $\left(\boldsymbol{\theta}_{\mathbf{4}}\right)$ & Uncertainty $(\boldsymbol{\theta})$ \\
\hline $\mathrm{P}(1)$ & 0.7488 & 0.1044 & 0.0468 & 0.0000 & 0.1000 \\
\hline $\mathrm{C}(2)$ & 0.3600 & 0.0000 & 0.3357 & 0.2043 & 0.1035 \\
\hline $\mathrm{S}(3)$ & 0.0000 & 0.2538 & 0.5427 & 0.1000 \\
\hline $\mathrm{J}(4)$ & 0.2889 & 0.4788 & 0.1323 & 0.0000 \\
\hline
\end{tabular}

As seen from D-S evidence synthesis theory (rule), the synthetic formulation of product quality characteristic (P) and process quality characteristic (C) for $1^{\text {st }}$ process is shown as Equation (11). The synthetic result is presented in Table 7. 
Table 7 . The synthetic result of product quality characteristic and process quality characteristic

\begin{tabular}{|c|c|c|c|c|c|}
\hline Characteristics & Key process $\left(\boldsymbol{\theta}_{\mathbf{1}}\right)$ & More improtant process $\left(\boldsymbol{\theta}_{\mathbf{2}}\right)$ & Improtant process $\left(\boldsymbol{\theta}_{\mathbf{3}}\right)$ & General process $\left(\boldsymbol{\theta}_{\mathbf{4}}\right)$ & Uncertainty \\
\hline $\mathrm{PC}(1)$ & $\mathbf{0 . 8 0 0 5}$ & 0.0220 & 0.1135 & 0.0430 \\
\hline $\mathrm{S}(3)$ & 0.0000 & 0.2538 & 0.5427 & 0.0210 \\
\hline $\mathrm{J}(4)$ & 0.2889 & 0.4788 & 0.1323 & 0.1000 \\
\hline
\end{tabular}

As seen from Table 7, the synthetic PC quality characteristic will continue synthesizing with safety quality haracteristic(S). The synthesis process will terminate until the last quality characteristic being synthesized, as in Table 8.

$$
\begin{aligned}
& K_{1}=m_{1}\left(\theta_{1}\right)\left(m_{2}\left(\theta_{2}\right)+m_{2}\left(\theta_{3}\right)+m_{2}\left(\theta_{4}\right)\right)+m_{1}\left(\theta_{2}\right)\left(m_{2}\left(\theta_{1}\right)+m_{2}\left(\theta_{3}\right)+m_{2}\left(\theta_{4}\right)\right)+ \\
& m_{1}\left(\theta_{3}\right)\left(m_{2}\left(\theta_{1}\right)+m_{2}\left(\theta_{2}\right)+m_{2}\left(\theta_{4}\right)\right)+m_{1}\left(\theta_{4}\right)\left(m_{2}\left(\theta_{1}\right)+m_{2}\left(\theta_{2}\right)+m_{2}\left(\theta_{3}\right)\right) \\
& m\left(\theta_{1}\right)=\frac{m_{1}\left(\theta_{1}\right) m_{2}\left(\theta_{1}\right)+m_{1}\left(\theta_{1}\right) m_{2}(\theta)+m_{2}\left(\theta_{1}\right) m_{1}(\theta)}{1-K_{1}} \\
& m\left(\theta_{2}\right)=\frac{m_{1}\left(\theta_{2}\right) m_{2}\left(\theta_{2}\right)+m_{1}\left(\theta_{2}\right) m_{2}(\theta)+m_{2}\left(\theta_{2}\right) m_{1}(\theta)}{1-K_{1}} \\
& m\left(\theta_{3}\right)=\frac{m_{1}\left(\theta_{3}\right) m_{2}\left(\theta_{3}\right)+m_{1}\left(\theta_{3}\right) m_{2}(\theta)+m_{2}\left(\theta_{3}\right) m_{1}(\theta)}{1-K_{1}} \\
& m\left(\theta_{4}\right)=\frac{m_{1}\left(\theta_{4}\right) m_{2}\left(\theta_{4}\right)+m_{1}\left(\theta_{4}\right) m_{2}(\theta)+m_{2}\left(\theta_{4}\right) m_{1}(\theta)}{1-K_{1}} \\
& m(\theta)=\frac{m_{1}(\theta) m_{2}(\theta)}{1-K_{1}}
\end{aligned}
$$

\begin{tabular}{|c|c|c|c|c|c|}
\hline Process No. & Key process $\left(\theta_{1}\right)$ & More improtant process $\left(\theta_{2}\right)$ & Improtant process $\left(\theta_{3}\right)$ & General process $\left(\theta_{4}\right)$ & Uncertainty \\
\hline 1 & 0.5159 & 0.1397 & 0.3232 & 0.0178 & 0.0034 \\
\hline 2 & 0.6212 & 0.1867 & 0.1299 & 0.0604 & 0.0017 \\
\hline 21 & 0.4718 & 0.3018 & 0.2243 & 0.0000 & 0.0021 \\
\hline 22 & 0.5012 & 0.3524 & 0.1108 & 0.0323 & 0.0033 \\
\hline 23 & 0.7426 & 0.1789 & 0.0773 & 0.0000 & 0.0012 \\
\hline 24 & 0.6737 & 0.2015 & 0.0991 & 0.0241 & 0.0016 \\
\hline 25 & 0.6675 & 0.3071 & 0.0229 & 0.0000 & 0.0025 \\
\hline 26 & 0.6022 & 0.2976 & 0.0972 & 0.0000 & 0.0030 \\
\hline 28 & 0.5817 & 0.3291 & 0.0873 & 0.0000 & 0.0019 \\
\hline 38 & 0.6432 & 0.2515 & 0.0056 & 0.0960 & 0.0037 \\
\hline 39 & 0.4971 & 0.3397 & 0.0825 & 0.0777 & 0.0030 \\
\hline
\end{tabular}

Table 8. Synthetic result of four quality characteristic for $1^{\text {st }}$ process

\begin{tabular}{|c|c|c|c|c|c|}
\hline Characteristics & Key process $\left(\boldsymbol{\theta}_{\mathbf{1}}\right)$ & More improtant process $\left(\boldsymbol{\theta}_{\mathbf{2}}\right)$ & Improtant process $\left(\boldsymbol{\theta}_{\mathbf{3}}\right)$ & General process $\left(\boldsymbol{\theta}_{\mathbf{4}}\right)$ & Uncertainty \\
\hline PCSJ $(1)$ & $\mathbf{0 . 5 1 5 9}^{* *}$ & 0.1397 & 0.3232 & 0.0178 & 0.0034 \\
\hline
\end{tabular}

As seen from Table $8,1^{\text {st }}$ process is identified as key process. The specific analysis procedure on other processes is not addressed because of the same analysis method as that on $1^{\text {st }}$ process. The identified key processes are presented in Table 9.

Table 9. Basic confidence of key processes of coal preparation process

The criticality degree $u j(1 \leq j \leq 17)$ of each quality characteristic is calculated by Equation (8). According to Pareto Principle, the cumulative sum of ratio for criticality degree is presented as Figure 3.

As seen in Figure 3, $9^{\text {th }}$ characteristic (Equipment failure rate) is the most important characteristic. The key characteristic includes seven characteristics, i.e., $9^{\text {th }}$ characteristic (Equipment failure rate), $7^{\text {th }}$ characteristic (Calorific value), $8^{\text {th }}$ characteristic (Quantity of having medium), $1^{\text {st }}$ characteristic (Ash), $10^{\text {th }}$ characteristic (Water spray quantity and hydraulic pressure of de-medium), $11^{\text {th }}$ characteristic (Qualified medium density) and $6^{\text {th }}$ characteristi c (Impurity content). 


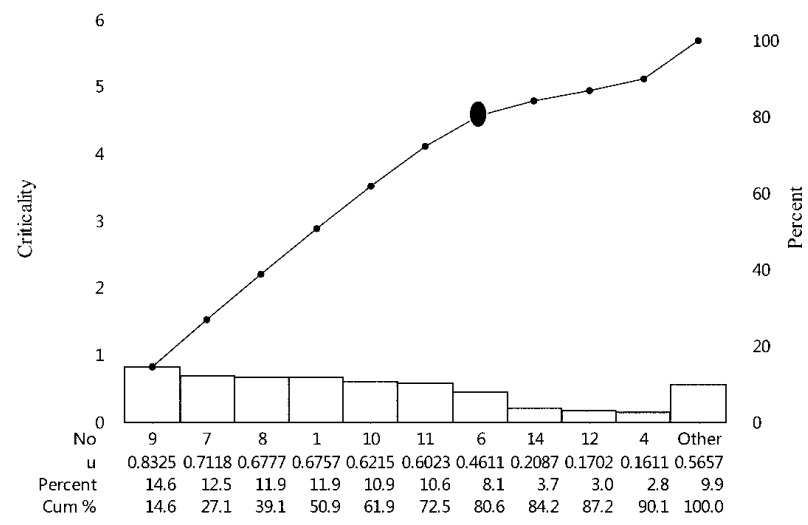

Figure 3. The Pareto diagram of criticality degree of quality characteristic

\subsection{Validity Analysis and Discussion}

\subsubsection{Validity Analysis of Proposed Approach}

In coal preparation system, the key processes identified by the proposed approach have significant impacts on quality characteristics from different dimensionality. It means the capability to reach the quality characteristic standards will be assured if the key process was monitored. Process capability analysis method is used to test whether the process is indeed the key process or not. The process is proposed. If the capability was improved after identification, analysis and control, we consider the proposed identification approach valid. Likewise, qualified medium density is identified as key quality characteristic. This characteristic plays an important role in processing of $22^{\text {th }}$ process (identified key process). The data about medium density of qualified medium bucket in dense medium workshop are collected. The data of dense medium are collected in two days before and after the control. According to the technical standards in this coal preparation plant, the qualified medium density in dense medium workshop is required at $1.7 \pm 0.02 \mathrm{~g} / \mathrm{cm}^{3}$. The process capability of density before and after identification and control is analyzed as shown in Figure 4.

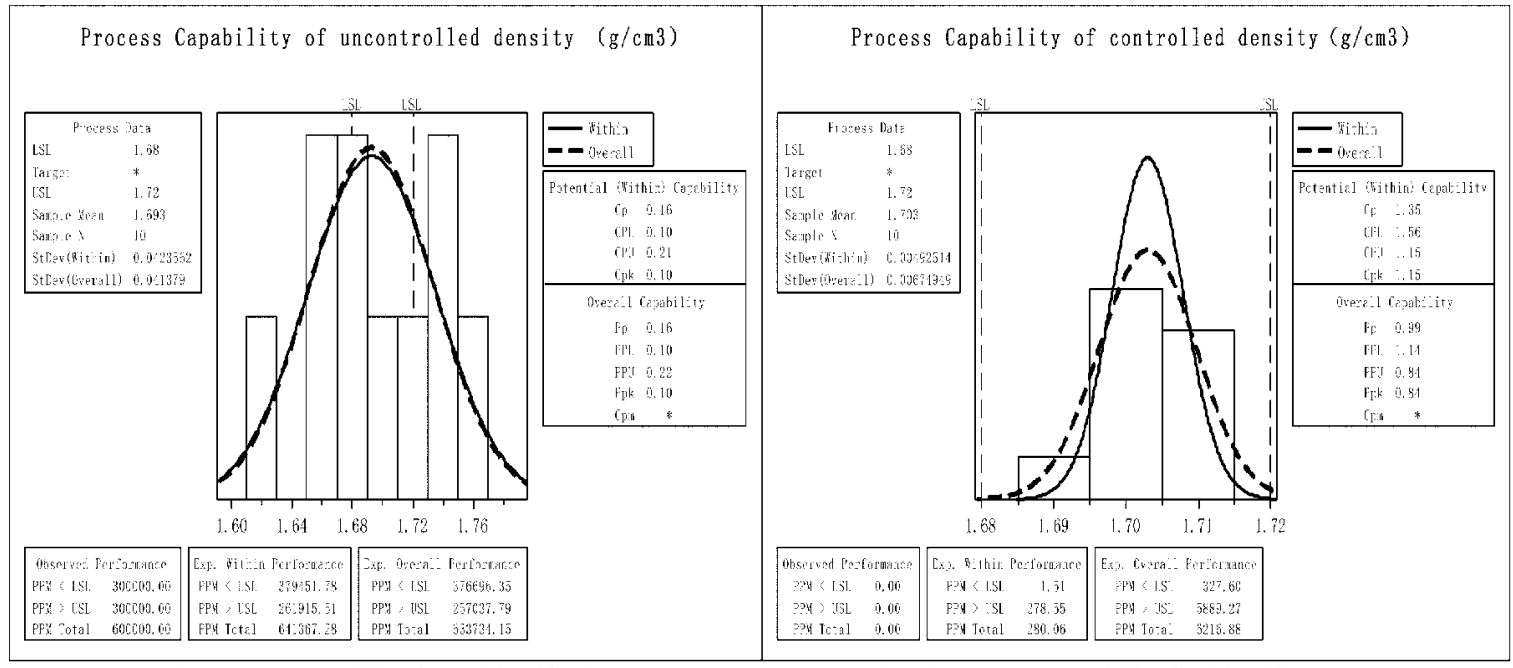

(a) The process capability for unidentified process

(b) The process capability for identified process

Figure 4. The process capability comparison

As shown in Figure 4, the process capability index (CPK) is 0.10 before the control, and the CPK is 1.15 after the control. The process capability is changed from grade D, which means unacceptable, to grade B, which means acceptable [42]. The process capability is improved, so that the proposed key processes and characteristics identification approach is considered to be valid.

\subsubsection{Discussion}

Consider the data of Table 6 and Table 8 that illustrate the initial belief value and the identification result for $1^{\text {st }}$ process, 
respectively. The process grade of $1^{\text {st }}$ process is the most possible key process based on product $(\mathrm{P})$ and process quality characteristics $(\mathrm{C})$. However, the process grade of $1^{\text {st }}$ process is identified as important and more important process based on safety (S) and work quality characteristics (J). Apparently, there are conflicts between these conclusions. This condition usually puzzles process personnel. After applying the principles of evidence theory, the belief function value of $\theta_{3}$ for $S(3)$ decrease to 0.3232 , while the belief function value of $\theta_{1}$ for $S(3)$ and $J(4)$ increase to 0.5159 . The belief function value of uncertain factors reduces to 0.0034 . The combined evidence enhances $\theta_{1}$ as the identified grade of $1^{\text {st }}$ process; therefore, the combined identification results solve the conflict between the conclusions derived from four evaluation indicators (quality characteristics), respectively and is thought to be more receivable than them.

In addition, EGM is used to acquire the raw data of correlation in this work. There are three subjects to evaluate to assure the effectiveness and reliability of data. Therefore, the evaluation result of three subjects is discussed in the next section. The final result of key characteristics identification has been given in Figure 3. Evaluated results of key characteristics from different subject are presented to discuss the similarities and differences. The independent and weighted criticality of various characteristics from three subjects is shown in Figure 5.

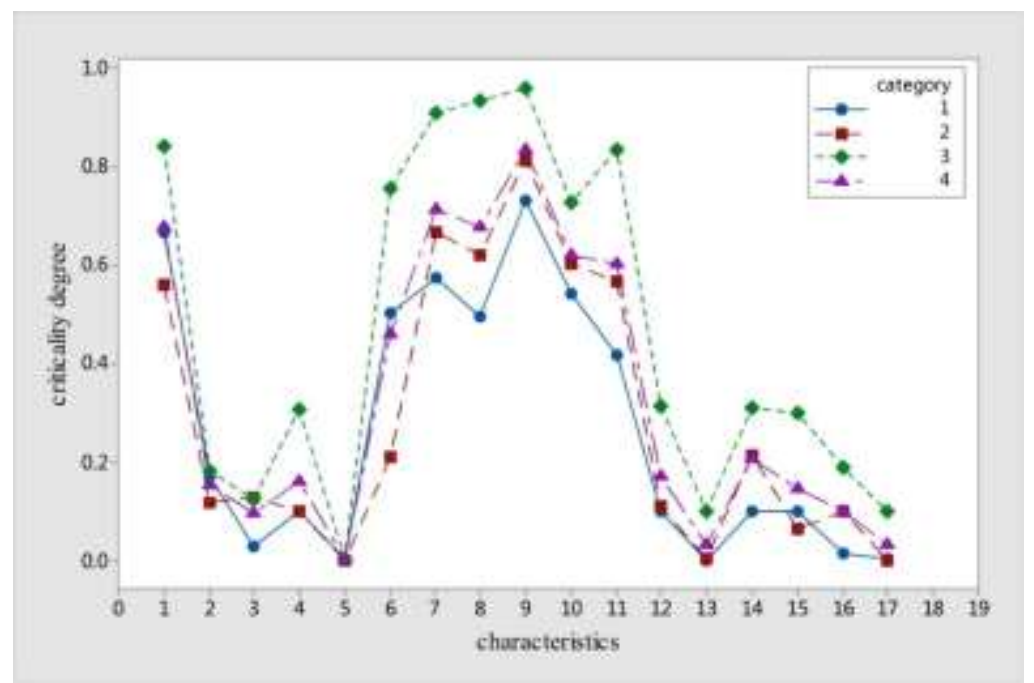

Figure 5. The scatter plot of criticality vs characteristics

In Figure 5, category 1 to category 4 represent senior manager, ordinary management personnel, operator and weighted criticality degree successively. As seen from Figure 5, there is a similar trend for curve of criticality degree. The $9^{\text {th }}$ characteristic has large criticality degree. The criticality degree from senior manager and ordinary management personnel is more similar than operator's. Besides, it can be seen that operator prefers to give a higher score. Operators on site believe $8^{\text {th }}$ characteristic is more important than $7^{\text {th }}$ characteristic while the other subjects insist that $7^{\text {th }}$ characteristic is more important. This is reasonable because the $7^{\text {th }}$ characteristic reflects the quality of final product, which is the focus of management personnel. However, operators on site always pay more attention to process variables to reduce defects of the product. The above discussion demonstrates the reliability of evaluation from three subjects.

Process can be divided into processing (a), transportation (b), inspection (c) and storage (d) by function. The statistics of function of identified processes is given in Table 10 .

\begin{tabular}{|c|c|c|}
\hline Type & Process No. & Statistics \\
\hline Key process & $1,2,21,22,23,24,25,26,28,38,39$ & $a(7), b(3), c(1)$ \\
\hline More important process & $3,5,6,8,11,19,30,34,16,27,45,48,53$ & $a(8), b(1), c(4)$ \\
\hline Important process & $4,7,15,17,18,29,33,37,46,49,50,51,52$ & $a(2), b(10), c(1)$ \\
\hline General process & $9,10,12,13,14,20,31,32,35,36,40,41,42,43,44,47$ & $b(9), c(2), d(5)$ \\
\hline
\end{tabular}

As seen from Table 10, most processes with processing function are identified key or important processes while all processes with storage function are treated as general processes. This shows that processes with processing function is crucial for shop-floor, which conforms to actual production condition. 


\section{Conclusions}

Focusing on the problem of key process and quality characteristic identification for complex manufacturing system, an integrated and systematic identification approach based on multi-source information fusion using dynamic weighting fuzzy function and evidence theory is proposed. Firstly, the modified casual matrix is used to construct the correlation between processes and quality characteristics. The corresponding data of correlation degree is provided based on experts scoring. Then, dynamic weighting function is proposed to assign the weight of various quality characteristics. Subsequently, triangular fuzzy function-based classification method is presented to give decision result of each process by independent quality characteristic, which may provide multiple different decision results. Finally, the D-S evidence synthesis rule successfully plays its intended role and makes final decision result by synthesizing multiple decisions. The key processes are identified by largest membership principle. The key quality characteristics are identified based on dynamic weighting score and Pareto Principle simultaneously. The performance of proposed approach is verified by real-time dense medium coal preparation process. Results show that the presented approach can solve the conflicts among multiple pieces of evidence (decision) derived from every quality characteristic. Meanwhile, we find the operators on site pay more attention to process quality characteristics and always give higher score of correlation. Moreover, the processes with processing function are generally considered more important than others. The key contributions of this research are as follows:

(i) A systematic framework of key processes and quality characteristics for sophisticated manufacturing system is provided in section 2.1. The framework consists of three aspects, viz. establishment of correlation and data acquisition, dynamic weighting of characteristics and specific identification method.

(ii) The key process identification approach is firstly proposed from quality vision. The data and expert knowledge based identification approach for key processes and quality characteristics are proposed to avoid the limitations of conventional methods.

(iii) The weight of quality characteristic is dynamic, which not only reflects the difference of various level of scoring criterion, but also shows the difference of the same level of scoring criterion.

(iv) D-S evidence synthesis rule-based decision-making method is presented to solve the problem of multiple decisions synthesis. Therefore, the final decision about key processes identification can reveal the comprehensive influence of each process on multiple quality characteristics.

(v) In this research work, the proposed approach can identify key processes and quality characteristics in the same framework, which avoids the individual research of each issue.

However, there are certain limitations of the proposed approach. The data used to evaluate and identify key processes and quality characteristics is based on expert experience and knowledge, which may reduce the accuracy of evaluation with the development of production system. The identification results cannot be given online. Besides, there may be large numbers of quality characteristic. Thus, the number of decision synthesis will be enormous while some characteristics may have little influence on process. Furthermore, the online identification approach based on industrial big data will be researched. The approach can mine the historical data and sensor data to derive the failure probability of each process. The key process can be identified by failure probability and quality loss function. Dimensionality reduction (e.g., Principle Component Analysis) or feature extraction method will be provided to reduce the workload of decision synthesis.

\section{Acknowledgements}

This research is supported by the National Natural Science Foundation of China (Grant No.71701113), Shandong Provincial Natural Science Foundation, China (Grant No. ZR2016GQ11), Project of Shandong Province Higher Educational Science and Technology Program (Grant No. J17KA167), and supported by SDUST Research Fund (Grant No.2018YQJH103).

\section{References}

1. K. J. Kim, "Determining Optimal Design Characteristic Levels in QFD,” Quality Engineering, Vol. 10, No. 2, pp. 295-307, 1997

2. Y. Sireli, P. Kauffmann, and E. Ozan, "Integration of Kano's Model into QFD for Multiple Product Design,” IEEE Transactions on Engineering Management, Vol. 54, No. 2, pp. 380-390, 2007

3. G. Tontini, "Identification of Customer Attractive and Must-be Requirements using a Modified Kano's Method: Guidelines and Case Study," American Quality Congress, 2000 
4. Z. Wang, R. Y. K. Fung, Y. L. Li, and Y. Pu, “An Integrated Decision-making Approach for Designing and Selecting Product Concepts based on QFD and Cumulative Prospect Theory," International Journal of Production Research, No. 7, pp. 1-16, 2017

5. W. Jia, Z. Liu, Z. Lin, C. Qiu, and J. Tan, "Quantification for the Importance Degree of Engineering Characteristics with a Multi-level Hierarchical Structure in QFD," International Journal of Production Research, Vol. 54, No. 6, pp. 1-23, 2016

6. T.Materla, E. A. Cudney, and J. Antony, "The Application of Kano Model in the Healthcare Industry: A Systematic Literature Review," Total Quality Management \& Business Excellence, Vol. 28, No. 7, 2017

7. G. Tontini, "Integrating the Kano Model and QFD for Designing New Products," Total Quality Management \& Business Excellence, Vol. 18, No. 6, pp. 599-612, 2007

8. Q. Xu, R. J. Jiao, X. Yang, M. Helander, H. M. Khalid, and A. Opperud, “An Analytical Kano Model for Customer Need Analysis," Design Studies, Vol. 30, No. 1, pp. 87-110, 2009

9. J. M. Kamil, P. J. Matthias, and M. W. Stephan, "Bottleneck Identification in Supply Chain Networks," International Journal of Production Research, Vol. 51, No. 5, pp. 1477-1490, 2013

10. A. Sujova, K. Marcinekova, and S. Hittmar, "Sustainable Optimization of Manufacturing Process Effectiveness in Furniture Production," Sustainability, Vol. 9, No. 6, pp. 923, 2017

11. S. Arumugam and J. Saral, "Identification of Bottleneck Elements in Cellular Manufacturing Problem," Procedia Computer Science, Vol. 74, pp. 181-185, 2015

12. C. Yu and A. Matta, "A Statistical Framework of Data-driven Bottleneck Identification in Manufacturing Systems," International Journal of Production Research, pp. 1-16, 2016

13. L. Li, Q. Chang, J. Ni, and B. Stephan, "Real Time Production Improvement through Bottleneck Control," International Journal of Production Research, Vol. 47, No. 21, pp. 6145-6158, 2009

14. S. 1. Sengupta, "Analysis of a New Signal for Bottleneck Identification and Loss Allocation to Individual Machines," International Journal of Industrial \& Systems Engineering, Vol. 13, No. 2, pp. 175-196, 2013

15. J. P. Loose, S. Zhou, and D. Ceglarek, "Variation Source Identification in Manufacturing Processes based on Relational Measurements of Key Product Characteristics," Journal of Manufacturing Science \& Engineering, Vol. 130, No. 3, pp. 361374,2008

16. X. Zhou and P. Jiang, "Variation Source Identification for Deep Hole Boring Process of Cutting-hard Workpiece based on Multi-source Information Fusion using Evidence Theory,” Journal of Intelligent Manufacturing, Vol. 28, pp. 1-16, 2014

17. Y. Wang and P. Jiang, "Fluctuation Evaluation and Identification Model for Small-batch Multistage Machining Processes of Complex Aircraft Parts," in Proceedings of the Institution of Mechanical Engineers Part B Journal of Engineering Manufacture, 2015

18. S. Du, R. Xu, D. Huang, and X. Yao, "Markov Modeling and Analysis of Multi-stage Manufacturing Systems with Remote Quality Information Feedback," Computers \& Industrial Engineering, Vol. 88, pp. 13-25, 2015

19. S. Du, R. Xu, and L. Li, "Modeling and Analysis of Multiproduct Multistage Manufacturing System for Quality Improvement," IEEE Transactions on Systems Man \& Cybernetics Systems, No. 99, pp. 1-20, 2016

20. M. Vandebroek, L. Lan, and K. Knapen, "An Experimental Diagnostic Procedure to Identify the Source of Defects in Multistage and Multi-component Production Processes," Journal of Quality Technology, Vol. 48, No. 3, pp. 213-226, 2016

21. M. Davoodi and S. T. A. Niaki, "Estimating the Step-change Time of the Location Parameter in Multistage Processes using MLE," Quality \& Reliability Engineering International, Vol. 28, No. 8, pp. 843-855, 2012

22. Y. Qin, L. Zhao, Y. Yao, and D. Xu, "Multistage Machining Processes Variation Propagation Analysis based on Machining Processes Weighted Network Performance," International Journal of Advanced Manufacturing Technology, Vol. 55, No. 5-8, pp. 487-499, 2011

23. S. Zhou, "Quality Control and Improvement for Multistage Systems: A Survey," IIE Transactions, Vol. 41, No. 9, pp. 744-753, 2009

24. S. Zhou, Y. Chen, and J. Shi, "Statistical Estimation and Testing for Variation Root-cause Identification of Multistage Manufacturing Processes," IEEE Transactions on Automation Science \& Engineering, Vol. 1, No. 1, pp.73-83, 2004

25. S. Zhou, Q. Huang, and J. Shi, "State Space Modeling of Dimensional Variation Propagation in Multistage Machining Process using Differential Motion Vectors," IEEE Transactions on Robotics \& Automation, Vol. 19, No. 2, pp. 296-309, 2003

26. R. Z. Tang, G. D.Wang, W. D. Tang, and S. Jia, "Key Process Identification Method for Workshop Quality Management," Journal of Zhejiang University (Engineering Science Edition), Vol. 46, No. 11, pp. 1937-1942, 2012

27. L. Ma, J. Mao, and H. Fan, "Key Quality Characteristics Identification Method for Mechanical Product," Procedia Cirp, Vol. 56, pp. 50-54, 2016

28. N. Wang, J. C. Xu, and J F Yang, "Identifying Method of Key Quality Characteristics in Multistage Manufacturing Process," 
Computer Integrated Manufacturing Systems, Vol. 217-219, No. 4, pp. 2580-2584, 2013

29. N. Jin, S. Zhou, T. S. Chan, and H. H. Huang, "Identification of Influential Functional Process Variables for Surface Quality Control in Hot Rolling Processes," IEEE Transactions on Automation Science \& Engineering, Vol. 5, No. 3, pp. 557-562, 2008

30. M. D. Foggia and D. M. D’Addona, "Identification of Critical Key Parameters and Their Impact to Zero-defect Manufacturing in the Investment Casting Process," Procedia Cirp, Vol. 12, No. 12, pp. 264-269, 2013

31. W. Li and L. Y. Zheng, "Process and Approaches of Identifying Key Characteristics in Conceptual Process Planning," Computer Integrated Manufacturing Systems, Vol. 13, No. 1, pp. 147-152, 2007

32. J. Kim, Q. Huang, and J. Shi, "Latent Variable based Key Process Variable Identification and Process Monitoring for Forging," Journal of Manufacturing Systems, Vol. 26, No. 1, pp. 53-61, 2007

33. Y. H. Ma, J. Lv, J. Mu, and Y. Zhang, "The Application Investigation of C\&E Matrix and FMEA in Setting Checkpoint of Aerospace Engineering Production," Quality and Reliability, No. 5, pp. 8-10, 2014

34. K. O. Cua, K. E. Mckone, and R. G. Schroeder, "Relationships Between Implementation of TQM, JIT, and TPM and Manufacturing Performance," Journal of Operations Management, Vol. 19, No. 6, pp. 675-694, 2001

35. D. I. Prajogo and A. S. Sohal, "TQM and Innovation: A Literature Review and Research Framework," Technovation, Vol. 21, No. 9, pp. 539-558, 2001

36. D. I. Prajogo and A. S. Sohal, "The Multidimensionality of TQM Practices in Determining Quality and Innovation Performance — An Empirical Examination,” Technovation, Vol. 24, No. 6, pp. 443-453, 2004

37. Z. B. Liu, Q. Wei, and X. H. Kong, "Fuzzy Comprehensive Evaluation on the Effect of Measures Operation for Oil-water Well," Hindawi Publishing Corp, 2011

38. J. Rosen, B. Hannaford, C. G. Richards, and M. N. Sinanan, "Markov Modeling of Minimally Invasive Surgery based on Tool/Tissue Interaction and Force/Torque Signatures for Evaluating Surgical Skills," IEEE Transactions on Biomedical Engineering, Vol. 48, No. 5, pp. 579-591, 2001

39. F. Wang, "Fusion Fingerprint and Face Multi-biometrics Recognition based on D-S Evidence Theory," Advanced Materials Research, Vol. 459, pp. 644-648, 2012

40. Y. Zhao, J. S. Mi, X. Liu, and X. Y. Sun, "Reconstructing Images Corrupted by Noise based on D-S Evidence Theory," International Journal of Machine Learning \& Cybernetics, pp. 1-8, 2015

41. H. J. Zhang, "Website Analysis: Website Management, Optimization, and Operations Driven by Data," China Machine, pp. 161-170, 2013

42. Z. Dou, "Research on Application of Process Capability Analysis in Manufacturing," Chinese Journal of Power Sources, Vol. 33, No. 10, pp. 896-898, 2009 